\section{La recepción del autor más citado y referenciado en el Congreso Mexicano de Investigación Educativa: Pierre Bourdieu}

The reception of the most cited and referenced author at the Mexican Congress of Educational Research: Pierre Bourdieu

Sandra Saraí Dimas Márquez

\section{RESUMEN}

Con el objetivo de brindar algunos hallazgos sobre la recepción de Pierre Bourdieu en el contexto mexicano, se revisaron ocho ediciones del Congreso Nacional de Investigación Educativa (del 2003 al 2017), lo anterior a través del análisis de las citas y referencias incluidas en las ponencias en donde apareció el autor. El estudio empleó herramientas metodológicas como el análisis de contenido y la estadística descriptiva para examinar las citas y referencias incluidas en las ponencias ya mencionadas. Dentro de los resultados, se pudo identificar que el sociólogo francés es el autor más citado y referenciado, además se pudo reconocer el papel que juega la cita y las obras referenciadas para considerar la popularidad del autor, de igual manera fue posible recuperar algunos indicios sobre la recepción de Bourdieu en México al leer al autor a través de otros, ya sea traductores o difusores. Finalmente, el estudio concluye en que la cita puede entenderse como una medida de consagración, como una condición de validez para un texto académico, como un factor de evaluación, como un objeto de lucha y como un capital cultural.

Palabras clave: producción científica; citas y referencias; Pierre Bourdieu

\section{ABSTRACT}

This study aims to show findings about Pierre Bourdieu's reception in Mexico, for this, it was analyzed 8 editions of the Education Research National Congress in Mexico from 2003 to 2017, through the analysis of the citations and references included in the papers where the author appeared. This study used methodological tools such as content analysis and descriptive statistics to examine the citations and references included in the papers. The results was obtained indicate that French sociologist is the most referenced author, so it was possible to recognize the role by the citation and the referenced works to consider the popularity of the author, in the same way, it was possible to recover some indications about the reception of Bourdieu in Mexico when reading the author through others, either translators or broadcasters. Finally, the study concludes that the quotation can be understood as a measure of consecration, as a condition for an academic writing, as a measure unit, as an evaluation criteria, as something that is truly worth fighting for, and as cultural capital.

Keywords: Scientific production; citations and references; Pierre Bourdieu
INFORMACIÓN:

http://doi.org/10.46652/runas.v2i4.51 ISSN $2737-6230$

Vol. 2, No. 4, 2021. e21051

Quito, Ecuador

Enviado: octubre 14, 2021

Aceptado: diciembre 03, 2021

Publicado: diciembre 12, 2021

Sección Dossier | Peer Reviewed

Publicación continua

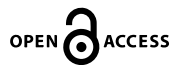

\section{(c) $(1)$}

AUTORA:

Sandra Saraí Dimas Márquez Universidad Autónoma del Estado de Hidalgo - México

sandra_dimas@uaeh.edu.mx

CONFLICTO DE INTERESES

El autor declara que no existe conflicto de interés posible.

FINANCIAMIENTO

No existió asistencia financiera de partes externas al presente artículo. NOTA

El artículo retoma hallazgos de la tesis doctoral: "Condiciones de recepción y consumo de la obra de Pierre Bourdieu en el Congreso Mexicano de Investigación Educativa".

PUBLISHER 


\section{Ideas introductorias}

El presente estudio se centró en los congresos como espacios académicos de difusión de prácticas científicas, atrayentes y cercanos a investigadores, académicos y estudiantes que buscan, algún tipo de reconocimiento en el campo científico con la intención de legitimar algún tipo de capital cultural y a su vez convertirlo otro u otros tipos de capitales (simbólico, social o económico).

Adicional a lo anterior, y bajo el amparo de la jerga bourdiana, los congresos académicos son espacios en donde confluyen diversos tipos de capital: capital cultural objetivado, institucionalizado e incorporado por parte de los participantes y de las instancias organizadoras; capital social, ya que el congreso se convierte en una oportunidad para establecer lazos, redes, comunidades académicas, entre otros; capital económico al ser un espacio que recauda fondos a través de las cuotas de los participantes y asistentes, y capital simbólico por el prestigio de cada congreso y por el reconocimiento a diversas figuras académicas. Y en el caso de los estudiantes o aspirantes a posgrado, los congresos se convierten en capital simbólico y cultural institucionalizado para el acceso, permanencia y egreso.

Se analizó el contenido a la producción científica objetivada en ponencias presentadas en 8 ediciones del Congreso Nacional de Investigación Educativa (ediciones llevadas a cabo en 2003, 2005, 2007, 2009, 2011, 2013, 2015 y 2017) con la finalidad de identificar el uso de la cita y la referencia, del autor más citado y referenciado en dicho congreso: Pierre Bourdieu.

Este estudio se enmarca en el análisis de la recepción de un autor y su obra, al respecto, Bourdieu (2015) afirma que la recepción, en tanto consumo cultural, depende de categorías de percepción y de apreciación de los receptores, mismas que están inscritas en las condiciones sociales de los mismos, ubicando una importante relación entre la producción y el consumo.

Cabe destacar que el análisis de la recepción de Pierre Bourdieu no es un asunto ajeno a la producción científica internacional, aunque sí para el caso mexicano, en un ejercicio de análisis documental, se realizó un estado del arte en donde se identificaron estudios de diversos países en donde el sociólogo francés ha sido objeto de reflexión respecto a su recepción, lo anterior resultado de la revisión de 28 trabajos (21 artículos, 2 capítulos de libro, 2 libros y 3 ponencias), que van del 2005 al 2016, publicados países de Europa, Asia, América y Oceanía.

De los trabajos revisados, se identificó que Argentina y Brasil son los que más interés mostraron en el estudio de la importación de ideas del sociólogo francés, lo anterior podría explicarse debido a la temprana importación y difusión del autor en estos contextos latinoamericanos, a través de estudiantes de posgrado que viajaron a Francia y que tuvieron contacto con el autor o con sus ideas.

De dichos trabajos, se recuperaron las unidades de análisis y objetos de reflexión, llama la atención la preponderancia de las obras, académicos, citas, traducciones, conceptos y referencias como elementos para detectar la recepción del autor; cabe aclarar que la mitad de los estudios emplearon solo una unidad de análisis, por ejemplo, Zavisca y Sallaz (2008) solo consideraron 
artículos de revista; Sapiro y Bustamante (2009) solo analizaron los libros del autor; y la otra mitad de los estudios empleó más de una unidad de análisis, tal es el caso de Chen y Zang (2009) que analizan libros del autor, tesis, artículos de revistas y ponencias; o el caso de Baranger (2008, 2011, 2013) que recupera planes de estudio, artículos de la ARSS, trayectorias de académicos y obras del autor.

Lo mismo ocurrió con los objetos de reflexión, estos entendidos como aquellos elementos focalizados en cada unidad de análisis, algunos consideraron solo uno y otros emplearon más de uno, por ejemplo, de los artículos de revistas se consideraron los conceptos y referencias contenidos en ellos (Zavisca y Sallaz, 2008); o bien las citas (Woodward \& Emmison, 2009); o las citas y los conceptos (Catani et al., 2005); de las obras del autor, se analizaron las traducciones (Bikbov, 2009; Filho, 2009; Gemperle, 2009); o en el caso de los que emplearon más de un objeto de reflexión, por ejemplo, Gelernter y Silber (2009) consideraron como unidades de análisis artículos de revistas, obras del autor y académicos, y como objetos de reflexión de dichas unidades, retomaron, traducciones, citas y datos de la trayectoria de los académicos.

De lo anterior, fue posible identificar que uno de los principales aportes de este estudio es el análisis de la recepción de Pierre Bourdieu en el contexto mexicano, pues a pesar de que algunos trabajos encontraron la presencia del autor en México, ninguno de ellos realizó un estudio sistematizando de la producción mexicana como el que se realizó con las ocho ediciones del Congreso Nacional de Investigación Educativa (CNIE).

Al respecto, Castro y Suárez (2018) señalan la necesidad de revisar de manera exhaustiva la presencia de Bourdieu en México "esa tarea se hizo con resultados muy fructíferos por distintos equipos en Argentina y Brasil, y en México es un esfuerzo pendiente (...) en suma, es una tarea pendiente emprender una sociología de la sociología de Bourdieu en México.” (p. 14).

De ahí que el objetivo del artículo es brindar los principales hallazgos del estudio a la recepción de Pierre Bourdieu en el Congreso Mexicano de Investigación Educativa a través del análisis de las citas y referencias (como unidades de reflexión), incluidas en las ponencias (como unidades de análisis) de las ya mencionadas ocho ediciones.

\section{Andamiajes metodológicos}

Como se señaló, en este estudio se revisaron las ponencias presentadas en los congresos que organiza el Consejo Mexicano de Investigación Educativa (COMIE), se eligieron estos congresos debido al prestigio que ha ido acumulando el COMIE a lo largo de su trayectoria, cabe señalar que este Consejo fue creado a partir de la consolidación de grupos de académicos, docentes, centros de investigación y distintas instituciones educativas, interesados entre otras cosas, en la producción y difusión científica. Entre las principales actividades académicas de dicho consejo destacan (COMIE, 2019): 
Los Congresos Nacionales de Investigación Educativa (CNIE), realizados cada dos años desde 1993. Y desde 2011, se abrió un espacio exclusivo para que los estudiantes de posgrado presenten los avances de sus investigaciones, denominado Encuentro Nacional de Estudiantes de Posgrado en Educación (ENEPE).

Publicación trimestral de Revista Mexicana de Investigación Educativa creada en 1996. Derivado de las investigaciones presentadas en las diversas ediciones del CNIE, el COMIE ha realizado en 2002 y en 2012, la publicación de los estados del conocimiento en torno a diferentes ejes temáticos del campo educativo.

Publicación de libros sobre temáticas del campo educativo.

En este sentido, se puede afirmar que los congresos del COMIE han acumulado determinado capital simbólico y social que los hace influyentes dentro del campo académico, particularmente dentro de la investigación educativa, además de que los congresos en tanto eventos científicos y académicos son espacios en los cuales es posible objetivar al sujeto objetivante, en este caso, es posible objetivar al autor de la ponencia, de ahí que, recuperando a Baranger (2011):

\section{[...] los congresos interesan por sí mismos en tanto funcionan como instancias de reproducción de las comunidades disciplinares, y también puede pensarse que las ponencias en ellos presentadas constituyen un corpus más próximo a la realidad cotidiana de las prácticas de investigación en el campo (las publicaciones en revistas en cualquier contexto siendo habitualmente más selectivas) (2011, p. 33).}

Se entiende que una ponencia es un tipo de comunicación científica que se difunde en una convención reconocida por una comunidad científica en la cual se plasman de manera concreta los resultados, avances o reflexiones de un trabajo de investigación, para Moyano (2001) la ponencia es "un texto de oralidad secundaria, en tanto que es escrito para ser expuesto oralmente o leído en voz alta en una mesa compartida con otros expositores en un congreso" (p. 7,8), generalmente cada congreso define tanto la extensión como los apartados de las mismas, los cuales casi siempre contemplan: título, resumen, palabras clave, introducción, objetivos, metodología, resultados, conclusiones y referencias.

Para llevar a cabo el análisis de la recepción de Bourdieu en el contexto mexicano, se compilaron las ponencias de ocho versiones del COMIE disponibles en la web, se recuperaron 6627 ponencias publicadas entre 2003 al 2017, se realizó un análisis de contenido y estadístico descriptivo tanto de las coincidencias presentadas al introducir "Bourdieu", "Bourdie" "Bordiu", en el lector de los archivos en formato PDF (formato en el cual se encuentran disponibles las ponencias en la web), como de diversos autores prominentes del campo educativo y del campo de la ciencia social, lo anterior para pensar a Bourdieu con relación a otros teóricos. Se tomaron dos criterios para la elección de los autores, el primero fue distinguir aquellos con mayor popularidad en el escenario actual, y el segundo considerar a los autores “clásicos" en cada campo.

Los autores elegidos del campo educativo fueron: Jean Piaget, Lev Vigotsky, John Dewey, Paulo Freire, Henry Giroux, Michael Apple, Peter Mc Laren; y del campo de la ciencia social: Karl Marx, 
Max Weber, Emile Durkheim, Michel Foucault, Alain Touraine, Jurgen Habermas, Norbert Elias, Niklas Luhmann, Anthony Giddens, Manuel Castells, Antonio Gramsci, Edgar Morin y Zygmunt Bauman.

Las herramientas metodológicas que amparan el análisis de la información son, por un lado, el análisis de contenido a las ponencias como unidades de análisis y la estadística descriptiva para detectar frecuencias en los objetos de reflexión, que en este caso fueron las citas y las referencias.

Para el caso del análisis de contenido, conviene señalar que en un primer momento se identificaron los autores antes descritos en cada ponencia, y en un segundo momento se recuperaron únicamente las ponencias que incluyeron la mención, cita y/o referencia del sociólogo francés: Pierre Bourdieu, de manera que se seleccionaron 521 ponencias como unidades analíticas.

Con respecto al análisis cuantitativo se recuperaron las herramientas de la estadística descriptiva, considerando las frecuencias de aparición de cada autor y posteriormente la frecuencia de las citas y referencias incorporadas en cada ponencia que incluyó a Bourdieu en sus referentes.

Para identificar el tipo de aparición de Pierre Bourdieu en cada ponencia, esto es, si el autor apareció en una cita, en una referencia, en una cita secundaria, en una cita y una referencia o si solo se mencionó, se consideraron las clasificaciones que brinda el formato de citación APA(2010), el cual alude que una cita se coloca dentro del cuerpo del texto y contiene breve información sobre los datos de la fuente, es decir, el apellido y año para las citas indirectas y secundarias, y el apellido, año y número de página o párrafo para las citas directas.

Cabe añadir que las citas directas también conocidas como textuales, son aquellas en las que se reproduce al pie de la letra el trabajo de otro autor, en este caso como ya se mencionaba, para APA (2010) se incluye el apellido paterno del autor, el año y la página o párrafo en donde se encuentra el fragmento de texto extraído. Las citas indirectas son aquellas que se emplean en la paráfrasis, es decir, cuando se interpreta o amplía la idea de algún autor en la cual se utilizan palabras distintas al texto original, en este caso se puede colocar únicamente el apellido del autor y el año. Respecto a las citas secundarias también llamadas citas de citas, son aquellas en las que se hace alusión a un autor citado en una fuente, por ejemplo: Bourdieu (citado en Giménez, 2004), APA recomienda emplear con moderación las fuentes secundarias y en la medida de lo posible citar siempre fuentes primarias.

Con relación a las referencias, en éstas se coloca la información completa de la obra y dicha información va al final del documento, aunque en otros formatos de citación la referencia puede incluirse al pie de página. Ahora bien, para el caso del estudio realizado, tuvo que agregarse un criterio más, el de la ‘mención' del autor, ya que hubo trabajos en los que se mencionaba al autor, pero no se incluía información de citas (en cualquiera de sus tipos) o en las referencias.

Cabe aclarar que en este escrito solo se ofrecen tablas y gráficos que permiten acercarse, desde una mirada provisoriamente cuantitativa, al análisis del consumo de la obra de Pierre Bourdieu en las ponencias presentadas en los congresos organizados por el Consejo Mexicano de Investigación Educativa realizados del 2003 al 2017. 


\section{Algunos resultados}

Para hablar de la recepción de una obra escrita, es decir, la forma en la que el lector se apropia de la obra del autor, es necesario, considerar múltiples factores, por ejemplo, Sapiro (2016) afirma que una vez que es publicada la obra, la recepción es mediatizada por las interpretaciones realizadas por instituciones y agentes que pertenezcan al campo intelectual.

¿Por qué Bourdieu? Como se mencionaba, Bourdieu fue el autor con más coincidencias en el análisis a 6627 ponencias publicadas entre 2003 al 2017 del CNIE, quien se formó en la filosofía, pero realizó estudios empíricos que contribuyeron a la antropología, la sociología, la educación, la historia, la lingüística, la ciencia, la política, la estética, la literatura, el derecho, entre otros, a partir de estudios sobre los campesinos, el parentesco, la familia, los deportes, los movimientos sociales, la práctica pedagógica, el trabajo, la pobreza, los consumos culturales, el arte, la fotografía, el género, el lenguaje, la vivienda, los académicos, el Estado, la religión, la opinión pública, la visita a los museos, la televisión, por mencionar algunos.

De modo que no es de extrañarse que un autor tan prolífico sea incluido en reportes de investigación para explicar y / o comprender fenómenos educativos. Lo que sí llama la atención es que Bourdieu se encuentra por encima de los clásicos de la educación, tal es el caso de Piaget, Vigotsky o Freire. Lo anterior puede apreciarse en el gráfico 1 y la tabla 1:

Gráfico 1. Hallazgos autores CNIE

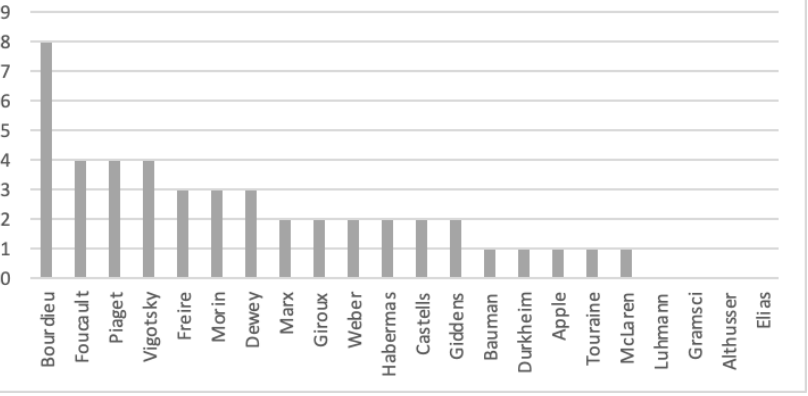

Fuente: Elaboración Propia a partir del análisis de frecuencias a los autores más citados en las ponencias revisadas

Tabla 1. Distribución de porcentajes por año

\begin{tabular}{|r|r|r|r|r|r|r|}
\hline Año & $\begin{array}{c}\text { Pierre } \\
\text { Bourdieu } \\
(\%)\end{array}$ & $\begin{array}{c}\text { Michel } \\
\text { Foucault } \\
(\%)\end{array}$ & $\begin{array}{c}\text { Jean Piaget } \\
(\%)\end{array}$ & $\begin{array}{c}\text { Lev } \\
\text { Vigotsky } \\
(\%)\end{array}$ & $\begin{array}{c}\text { Paulo } \\
\text { Freire (\%) }\end{array}$ & $\begin{array}{c}\text { Total de } \\
\text { ponencias } \\
(\mathbf{n})\end{array}$ \\
\hline 2003 & 8 & 4 & 6 & 6 & 1 & 269 \\
\hline 2005 & 8 & 4 & 4 & 2 & 4 & 431 \\
\hline 2007 & 10 & 4 & 4 & 3 & 2 & 699 \\
\hline 2009 & 10 & 6 & 4 & 5 & 3 & 849 \\
\hline 2011 & 8 & 4 & 4 & 3 & 4 & 937 \\
\hline 2013 & 8 & 3 & 3 & 3 & 4 & 1021 \\
\hline 2015 & 4 & 2 & 2 & 3 & 3 & 1238 \\
\hline 2017 & 8 & 5 & 4 & 5 & 5 & 1178 \\
\hline
\end{tabular}

Fuente: Elaboración Propia a partir del análisis de frecuencias a los autores más citados en las ponencias revisadas 


\subsection{Las citas}

Partiendo de la idea, de que la cita en tiempos actuales es además de un requisito en un documento académico, también se convierte en un signo de distinción, debido a que a través de ésta es posible sustentar algún argumento o idea, en este sentido, desde el planteamiento bourdiano la cita es un capital cultural incorporado que se objetiva en un escrito académico.

En el gráfico 2 es posible observar que poco más del 70\%, Bourdieu es citado y referenciado, mientras que el $30 \%$ restante corresponde a categorías que no se ajustan a los criterios definidos por el formato de citación solicitado en las ponencias, al respecto cabe considerar la función negligente que alude Bourdieu (2015) sobre todo para la categoría "cita secundaria” ya que ésta objetiva el desconocimiento del autor y su recepción a través de otros y no de las fuentes primarias.

Asimismo, se identificaron ponencias en donde solo aparecía la cita sin su correspondiente referencia como lo prescribe APA en la 6ta edición, otras en donde se incluyó solo la referencia sin incluir la cita, algunas en donde no se empleó ningún formato de citación y solo se hizo mención del autor.

En el contexto del texto, es decir, en el contenido de las ponencias que incluyen a las citas secundarias, solo citas, menciones y referencias, se identificaron escritos en donde hay un uso incidental del autor, esto es, un bajo dominio del autor respecto al uso de sus conceptos, y a pesar de citarlo, referenciarlo o mencionarlo, no da cuentan de la presencia de Bourdieu dentro de las ideas plasmadas en el texto.

Gráfico 2. Citas.

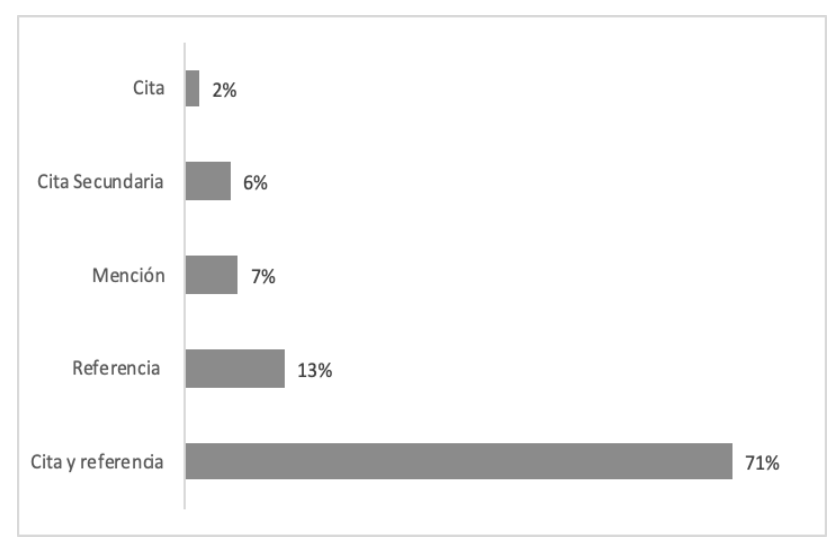

Fuente: Elaboración Propia a partir del análisis de frecuencias a los autores más citados en las ponencias revisadas

\subsection{Las obras referenciadas}

Para el caso de las obras referenciadas, vale la pena pensar brevemente tanto en el papel de las traducciones a la lengua hispana como en el papel de los difusores o importadores de las ideas bourdianas al contexto mexicano. Para el caso de las traducciones, Afranio García (en Sorá, 
2003) señala que la traducción deviene de una forma de consagración de un autor, y que el acto de traducir implica de algún modo, apropiarse de una referencia constituida a partir de otros presupuestos culturales, en este sentido, la traducción es un hecho social, cultural e histórico.

La primera obra de Bourdieu traducida al español fue Campo intelectual y proyecto creador, por la editorial siglo XXI, en 1967 tan solo un año después de su publicación en su idioma original, sin embargo, el libro Sociología de Argelia tardó 48 años en traducirse al idioma español, lo anterior brinda un referente para pensar que la lectura de la obra de Pierre Bourdieu, en países de habla hispana llegó de manera fragmentada, lo cual, de acuerdo con Mauger (2002) puede representar un obstáculo entre el texto y el contexto en la importación de ideas de Bourdieu.

Ahora bien, para el caso de los difusores o importadores de las ideas bourdianas en el contexto mexicano, es posible identificar el papel de diversos autores (en su mayoría extranjeros) que, como ya se mencionaba, tuvieron, contacto con el autor o sus ideas en el contexto académico francés, está el caso de Néstor García Canclini, Emilio Tenti Fanfani, Gilberto Giménez, Hugo José Suarez, por mencionar algunos, quien desde su posición como formadores, fueron difundiendo la obra de Bourdieu en el contexto académico mexicano.

En seguida, se agrega una tabla que muestra que más de la mitad del total de las ponencias analizadas solo emplea una obra del autor, en tanto que el resto emplea ninguna o hasta seis obras, estos últimos fueron catalogados como aquellos que presentan un mayor dominio del autor.

A este respecto, podría pensarse que un ponente con un alto dominio del autor, podría incluir más de dos obras del sociólogo francés, valga señalar que la obra de Bourdieu no se agota en una sola obra, sino que para entenderlo, es necesario conocer más de una obra, de lo contrario se corre el peligro de hacer lecturas erróneas o bien, quedarse con algunos planteamientos aislados que el autor sugirió en el algún momento de su trayectoria, o quedarse con interpretaciones a "primera vista" de conceptos o nociones, por ejemplo, la noción de habitus tiene un significado diferente en La Reproducción (donde refiere que el habitus era entendido como un asunto capaz de perpetuarse o como una disposición irreversible) que el El sentido práctico, o que en Respuestas por una antropología reflexiva/Una invitación a la sociología reflexiva y en La miseria del mundo donde plantea formas de modificar el habitus.

Tabla 2. de obras citadas CNIE.

\begin{tabular}{|l|c|}
\hline \multicolumn{2}{|c|}{ Número de obras citadas en el } \\
\hline Una obra & $\%$ \\
\hline Ninguna obra & 56 \\
\hline Dos obras & 16 \\
\hline Tres obras & 16 \\
\hline Cuatro obras & 7 \\
\hline Cinco obras & 2 \\
\hline Seis obras & 1 \\
\hline
\end{tabular}

Fuente: Elaboración Propia a partir del análisis de frecuencias a las obras más citadas en las ponencias revisadas 
En complemento con lo anterior, de las obras referenciadas de Bourdieu en las ponencias que se analizaron, se encuentran, en su mayoría, aquellas que fueron publicadas su idioma original y/o traducidas al español en la época en la que Pierre Bourdieu adquirió mayor fama a nivel internacional, es decir, en la década de los 9o's, en la tabla 3, puede identificarse la diferencia de años en traducción, al respecto, Sapiro y Bustamante (2009) señalan que una de las condiciones en el análisis de la recepción del autor, es el tiempo de traducción, ya que este indicador permite identificar la popularidad del autor de determinados espacios geográficos, de modo que lo que se muestra en la tabla puede resultar un importante indicador para pensar en la recepción de Bourdieu en el contexto educativo mexicano en al menos las dos primeras décadas del siglo XXI.

Tabla 3. Obras citadas CNIE.

\begin{tabular}{|c|c|c|c|c|}
\hline Título en español & $\%$ COMIE & $\begin{array}{l}\text { Año } \\
\text { (idioma } \\
\text { original) }\end{array}$ & $\begin{array}{l}\text { Año } \\
\text { (español) } \\
\text { 1era edición }\end{array}$ & $\begin{array}{l}\text { Diferencia } \\
\text { de años de } \\
\text { traducción }\end{array}$ \\
\hline Razones prácticas & 12 & 1994 & 1997 & 3 \\
\hline La reproducción & 12 & 1970 & 1977 & 7 \\
\hline Sociología y cultura* & 10 & 1981 & 1990 & 9 \\
\hline Capital cultural, escuela y espacio social* & 10 & 1997 & 1997 & 0 \\
\hline Respuestas por una antropología reflexiva** & 10 & 1992 & 1995 & 3 \\
\hline La distinción & 9 & 1979 & 1988 & 9 \\
\hline El sentido práctico & 9 & 1980 & 1990 & 10 \\
\hline El oficio de sociólogo & 7 & 1968 & 1975 & 7 \\
\hline Los Tres Estados del Capital Cultural*** & 6 & 1979 & 1987 & 8 \\
\hline Los herederos & 5 & 1964 & 1967 & 3 \\
\hline Cosas dichas & 5 & 1987 & 1988 & 1 \\
\hline El oficio del cientîfico & 4 & 2001 & 2003 & 2 \\
\hline Los usos sociales de la Ciencia & 4 & 1997 & 1999 & 2 \\
\hline La miseria del mundo & 4 & 1993 & 1999 & 6 \\
\hline La dominación masculina & 3 & 1998 & 2000 & 2 \\
\hline Homo academicus & 2 & 1984 & 2009 & 25 \\
\hline Una invitación a la sociología reflexiva** & 2 & 1992 & 2005 & 13 \\
\hline \multicolumn{5}{|c|}{$\begin{array}{l}\text { * Obras compiladas con contribuciones publicadas en su idioma original en diferentes años } \\
\text { **Misma obra con traducciones al español en diferentes momentos y de diferentes idiomas, la primera del francés al español y la segunda del } \\
\text { inglés al español. }\end{array}$} \\
\hline
\end{tabular}

Fuente: Elaboración Propia a partir del análisis de frecuencias a las obras más citadas en las ponencias revisadas

\section{Sobre los resultados, algunas discusiones}

En principio de cuentas conviene pensar en la cita como un objeto de reflexión, ya que ésta además de ser "una medida de consagración internacional” (Sapiro, 2017, p. 43), también permite detectar el crédito que hace el consumidor al productor, asimismo se puede pensar a la cita como una forma una condición de validez, argumentación y fiabilidad de una idea puesta en un texto académico; la cita es a su vez, un requisito de legitimidad, y para instancias evaluadoras de la producción científica en tiempos actuales, también puede pensarse como una unidad de medida, un factor de evaluación, y un objeto de lucha en la competencia por la legitimidad, lo anterior en el marco de un campo académico, científico e intelectual. A este respecto, Bourdieu (2012) afirma que: 
En el intercambio científico, el sabio aporta una «contribución» que le es reconocida por unos actos de reconocimiento público, por ejemplo, la referencia en forma de cita de las fuentes del conocimiento utilizado. Equivale a decir que el capital científico es el producto del reconocimiento de los competidores (un acto de reconocimiento que aporta tanto más capital cuanto más reconocido sea el que lo realiza, y, por consiguiente, más autónomo y con mayor capital) (Bourdieu, 2012, pp.100-101).

Para el caso de las ponencias que se analizaron en las ocho ediciones del CNIE, las citas y las referencias fueron requisitos de contenido por parte del congreso, sin embargo, la rigurosidad respecto a los formatos de citación inició a partir de la décima edición del congreso llevado a cabo en 2009, vale mencionar que a lo largo de las convocatorias emitidas de congresos estudiados, se identificaron cambios y ajustes a distintos criterios de los productos concursables (ponencias, carteles, libros, talleres, etc.) y lo que respecta al formato de citación en los últimos años, se especificó como APA (6ta edición), afianzando así el CNIE, a lo largo de su trayectoria sus normas de producción, lo cual le ha permitido funcionar como un mercado específico en el campo de la investigación en México, en el cual la “cita” está dotada de valor en la economía propia del campo.

A este respecto, para Bourdieu (2015) el campo de la producción y circulación de bienes simbólicos es definido como un sistema de relaciones entre la producción, la reproducción y la difusión de dichos bienes. Para el caso específico de la producción, el autor señala que este campo se estructura por dos principios: el primero es entre la oposición del campo de la producción restringida y el gran campo de la producción simbólica, y el segundo por la relación de oposición y complementariedad que se da entre el campo de producción restringida y las instancias de conservación, lo cual a su vez tiene impacto en la estructuración del campo global entre la producción y la circulación de bienes simbólicos.

Ahora bien, respecto al campo de la producción restringida, entendido como aquel campo que produce para los productores, éste funciona como un mercado cuya finalidad es la competencia por la legitimidad cultural en donde las técnicas o estilos que funcionan al interior de ese campo están dotados de valor, lo anterior debido a que éstos pueden conferir a los productores un valor, el cual es susceptible de ser percibido y reconocido por sus pares, que son al mismo tiempo clientes, competidores y consumidores (Bourdieu, 2015).

De esta manera, el campo de la producción restringida tiende a establecer sus normas de producción, los criterios de evaluación y en él se compite por el reconocimiento otorgado por el grupo de pares, lo cual hace que también se impongan los límites que condenan "todo recurso técnicamente acorde a procedimientos de distinción no reconocidos” (Bourdieu, 2015, p.94).

Por tanto, es entendible que por ejemplo en la XIV edición del CNIE-2017, de las 2337 ponencias registradas, 1183 fueron aceptadas y 1154 rechazadas (COMIE, 2017) esto es casi el 50\%. Cabe mencionar que las ponencias son evaluadas a doble ciego, esto significa que los dictaminadores de cada ponencia son dos expertos en la materia y que desconocen la identidad del autor o autores del escrito. 
De modo que, si se piensa a la cita como un objeto de lucha en la competencia por la legitimidad, de reconocimiento o bien como un capital cultural poseído por quien escribe (tanto del formato de citación, como del conocimiento o apropiación del autor que cita), es posible develar grosso modo la diversidad de "valores" en la economía propia del campo de la producción científica del CNIE. Esto significa que la gente cita, no solo por requisito sino también para asignarle un valor legítimo a la obra que produce (al texto científico o académico), lo cual a su vez puede interpretarse como la puesta en juego de una estrategia cuya apuesta es la conquista de la legitimidad cultural.

En el gráfico 2, se observó un gran porcentaje de ponentes citaron y referenciaron, sin embargo, los que no lo hicieron ofrecieron indicios de categorías que no se ajustan a los criterios definidos por el formato de citación solicitado en las ponencias, pero que su aparición permitieron considerar dos polos importantes, el primero de ellos, la función negligente que alude Bourdieu (2015) sobre todo para la categoría "cita secundaria” ya que ésta objetiva el desconocimiento del autor y su recepción a través de otros y no de las fuentes primarias, el otro polo podría dar cuenta de lo implícito del conocimiento del autor al solo mencionarlo, solo citarlo o solo referenciarlo, sin embargo, al profundizar en el contenido de las ponencias sobre el uso que le dan al autor, se ubicaron textos con Bourdieu y también se identificó a Bourdieu sin textos, esto es, textos cuyos planteamientos dan cuenta del uso de conceptos relacionales como herramientas y escritos en donde hay un uso incidental y a pesar de citarlo, referenciarlo o mencionarlo, no da cuentan de la presencia de Bourdieu dentro de las ideas plasmadas en el texto.

Por otra parte, respecto a las obras, en la tabla 3, se observan los resultados de las obras incluidas en las ponencias, los cuales invitan a reflexionar respecto a las obras más famosas en el escenario latinoamericano: "La reproducción” y "El oficio de sociólogo", valga señalar que los primeros libros que circularon en América Latina fueron los ya mencionados junto con "Los Herederos" (los tres en coautoría con Jean-Claude Passeron). No obstante, en el contexto mexicano y argentino (respectivamente) tomaron mayor relevancia tanto "La reproducción" como "El oficio de sociólogo" (Baranger, 2008; 2013)

Así, en el escenario mexicano "La Reproducción” se convirtió en un texto altamente difundido, sobre todo con la llegada a México de Emilio Tenti Fanfani, quien introdujo esta lectura para la docencia y la investigación en el campo educativo. “En España y en México, Bourdieu fue conocido primero y durante largo tiempo fundamentalmente en el campo de las ciencias de la educación" (Martínez, 2007, p.17).

Con relación a lo anterior, Bourdieu invita a pensar en los diferentes tipos de lecturas que se hacen a partir de los espacios diferenciados "No digo la misma cosa a los judíos de New York, de París, de Berlín o de Jerusalem y sin embargo no miento jamás” (Bourdieu, 1999, p. 198).

Las estructuras de los campos intelectuales nacionales actúan, como una mediación cultural en el comercio internacional de ideas: las estructuras del país exportador dan forma al producto y las del país receptor actúan como una especie de prisma mental que selecciona y refracta las ideas que encuentran un eco en su propia configuración y las retraducen al idioma teórico local 
(Mauger, 2007). Adicional a lo planteado, en la tabla 2 se identificó la lectura fragmentada a las obras del autor, ya que, como se señalaba, la obra de Bourdieu es posible comprenderla no solo desde una referencia, sino que todo su arsenal teórico se encuentra plasmado en distintas obras, por lo que habrá que considerar el import- export intelectual en la circulación internacional de ideas, y el papel de los traductores, mediadores, como elementos influyentes en el consumo de una obra (Mauger, 2007).

\section{Conclusiones provisorias.}

En escenarios actuales, el campo académico - intelectual exige a sus jugadores contar con determinados capitales que les permitan jugar en el juego de la producción científica, en este sentido, es posible afirmar que el campo académico, intelectual y científico, es por excelencia un campo de luchas, en donde el homo academicus disputa por distintas formas de reconocimiento que se encauzan en un reconocimiento de tipo simbólico.

En este estudio, se recuperó uno de esos tipos de capital que el agente posee y que invierte y reconvierte en el campo de la producción científica, el capital cultural, entendido como aquellos insumos teóricos - metodológicos con los que los jugadores miran sus objetos de estudio o tratan de explicar o comprender determinados fenómenos, por lo que frases como: "de acuerdo con”, "en un estudio realizado por”, “según diversos autores”, se convirtieron en objetos de reflexión. Cabe señalar que la ponencia es la objetivación de una toma de posición, y que, dentro del universo de autores propios de la educación o las ciencias sociales, fue Bourdieu el que más emplearon para analizar sus objetos de estudio.

Pierre Bourdieu como un nombre propio, cuya designación se inserta en el campo de los discursos científicos de la sociología, es también una nombre de "marca" que se ha recuperado o "apropiado" para "validar" discursos de otros en los campos de producción científica como es el caso de lo educativo, esto significa que es Bourdieu un nombre dotado de capital simbólico, una referencia internacional, lo cual puede ser una relativa ventaja, pues permite acercar sus nociones, conceptos y herramientas a diversas áreas de estudio, sin embargo, este conocimiento puede tener fuertes implicaciones en el desconocimiento de su obra, cayendo en riesgos de lecturas sustancialistas o por el contrario, hacer lecturas ultraortodoxas que limiten el uso creativo de sus conceptos como herramientas que permiten el análisis de objetos empíricos.

Leer a Bourdieu en México, como seguramente puede ocurrir en otros países de habla hispana, es leer al autor a través de otros (recuérdese el papel del traductor, el difusor y las lecturas fragmentadas) lo cual invita a pensar en el cuidado que ha de tenerse al emplear sus conceptos como medios legitimadores de propios discursos, ya que "si se parte de la hipótesis de que la gente no se lee, se comprenden un cúmulo de cosas que no se comprenden cuando se cree que se leen" (Bourdieu, 1999, pp. 201-202).

Si bien, en este artículo, solo se mostró una parte del análisis al consumo y recepción del autor (el uso de citas y referencias), dicho análisis invita a reflexionar con profundidad el fenómeno 
analizado y, hablando bourdianamente, es preciso considerar las condiciones objetivas y subjetivas que enmarcan la toma de posición del ponente ante el uso y la elección de Pierre Bourdieu frente a otros.

\section{Referencias}

APA. (2010). Manual de Publicaciones de la American Psychological Association. Manual Moderno.

Baranger, D. (2008). The Reception of Bourdieu in Latin America and Argentina. Sociologica, Italian journal of sociology, 2. https://www.rivisteweb.it/doi/10.2383/27724

Baranger, D. (2011). Antropología social y sociología argentinas: Identidades disciplinares en cuatro congresos. Revista Latinoamericana de Metodología de las Ciencias Sociales, 1(2), 23-59. https://www. relmecs.fahce.unlp.edu.ar/article/view/vo1nozao3

Baranger, D. (2013). La obra de Pierre Bourdieu y su recepción en Latinoamérica. En Á. Moreno, \& J. E. Ramírez. Pierre Bourdieu. Proyección Siglo XXI (pp. 157-194). Instituto Latinoamericano de Altos Estudios; Ambassade de France en Colombie.

Bikbov, A. (2009). A Strange Defeat: The Reception of Pierre Bourdieu's Works in Russia. Sociologica, Italian journal of sociology, 2. https://www.rivisteweb.it/doi/10.2383/31371

Bourdieu, P. (1999). Intelectuales, política y poder. Eudeba

Bourdieu, P. (2012). Los usos sociales de la ciencia. Nueva Visión

Bourdieu, P. (2015). El sentido social del gusto. Siglo XXI

Castro, R., \& Suárez, H. (2018). Introducción: trabajar con Pierre Bourdieu. En R. Castro, \& H. Suárez, Pierre Bourdieu en la Sociología Latinoamericana. El uso de Campo y habitus en la investigación (pp. 11-24). UNAM.

Catani, A., Catani, D., \& Gilson, R. (2005). Pierre Bourdieu: las lecturas de su obra en el campo educativo brasileño. Fundamentos en Humanidades, 6(12), 195-222. https://www.redalyc.org/pdf/184/18412611.pdf

Chen, N., \& Zang, X. (2009). Bourdieu and Chinese Sociology. Sociologica, Italian journal of sociology, 1. https:// www.rivisteweb.it/doi/10.2383/29570

COMIE. (2017). Numeralia del Congreso. Revista Mexicana de Investigación Educativa, 22(75), 1323-1330. http:// www.comie.org.mx/revista/v2018/rmie/index.php/nrmie/article/view/45

COMIE. (2019). Consejo Mexicano de Investigación Educativa A.C. COMIE. http://www.comie.org.mx/v5/ sitio/historia/

Filho, F. A. (2009). The Renovation: Aspects of Pierre Bourdieu's Reception in Brazil. Sociologica, Italian journal of sociology, 1. https://www.rivisteweb.it/doi/10.2383/29574

Gelernter, L., \& Silber, I. F. (2009). Bourdieu's Reception in Israeli Sociology. The Fragmented Imprint of a Grand Theory. Sociologica, Italian journal of sociology, 1. https://www.rivisteweb.it/doi/10.2383/29572

Gemperle, M. (2009). The Double Character of the German 'Bourdieu'. On the Twofold Use of Pierre Bourdieu's Work in the German-speaking Social Sciences. Sociologica, Italian journal of sociology, 2. https://www.rivisteweb.it/doi/10.2383/29573

Martínez, A. T. (2007). Lecturas y lectores de Bourdieu en la Argentina. Prismas - Revista de Historia Intelectual, 11(1), 11-30. https://www.redalyc.org/pdf/3870/387036796001.pdf 
Mauger, G. (2002). Sobre algunos obstáculos sociales a la comprensión de la obra de Pierre Bourdieu. In L.

E. Alonso, E. Martín-Criado, \& J. L. Moreno, Pierre Bourdieu, las herramientas del sociólogo (pp. 283-297). Editorial Fundamentos.

Mauger, G. (2007). Resistencias a la sociología de Pierre Bourdieu. In P. Champagne, L. Pinto, \& G. Sapiro, Pierre Bourdieu sociólogo (pp. 262-290). Nueva Visión

Moyano, E. I. (2001, mayo 2-5). Una clasificación de géneros científicos. Perspectivas Recientes sobre el discurso. [Presentación de ponencia] XIX Congreso AESLA, España. https://cutt.ly/JYRzHF8

Sapiro, G. (2016). La sociología de la literatura. Fondo de Cultura Económica.

Sapiro, G. (2017). Las condiciones de producción y circulación de bienes simbólicos. Instituto Mora.

Sapiro, G., \& Bustamante, M. (2009). Translation as a Measure of International Consecration. Mapping the World Distribution of Bourdieu's Books in Translation. Sociologica, Italian journal of sociology, 1. https:// www.rivisteweb.it/doi/10.2383/31374

Sorá, G. (2003). Traducir el Brasil. Una Antropología de la circulación internacional de ideas. Zorzal.

Woodward, I., \& Emmison, M. (2009). The Intellectual Reception of Bourdieu in Australian Social Sciences and Humanities. Sociologica, Italian journal of sociology, 1. https://www.rivisteweb.it/doi/10.2383/31370

Zavisca, J., \& Sallaz, J. (2008). From the Margins to the Mainstream. The Curious Convergence of Pierre Bourdieu and US Sociology. Sociologica, Italian journal of sociology, 2. https://www.rivisteweb.it/ doi/10.2383/27721

\section{AUTORA}

Sandra Saraí Dimas Márquez. Doctora en Ciencias Sociales, Maestra y Licenciada en Ciencias de la educación, por la Universidad Autónoma del Estado de Hidalgo docente y académica de la misma universidad en programas de maestría y para la formación docente. 